\title{
Minimising Unnecessary Mastectomies in a Predominantly Chinese Community
}

\author{
Mona P. Tan, ${ }^{1}$ Nadya Y. Sitoh, ${ }^{2}$ and Yih Y. Sitoh ${ }^{3}$ \\ ${ }^{1}$ Breast Surgical Oncology, MammoCare, 38 Irrawaddy No. 06-21, Singapore 329563 \\ ${ }^{2}$ MammoCare, 38 Irrawaddy No. 06-21, Singapore 329563 \\ ${ }^{3}$ Medical Education, Mount Elizabeth Hospital, 3 Mount Elizabeth No. 17-16, Singapore 228510 \\ Correspondence should be addressed to Mona P. Tan; jabezhopems@gmail.com
}

Received 14 September 2014; Accepted 2 January 2015

Academic Editor: C. H. Yip

Copyright (C) 2015 Mona P. Tan et al. This is an open access article distributed under the Creative Commons Attribution License, which permits unrestricted use, distribution, and reproduction in any medium, provided the original work is properly cited.

\begin{abstract}
Background. Recent data shows that the use of breast conservation treatment (BCT) for breast cancer may result in superior outcomes when compared with mastectomy. However, reported rates of BCT in predominantly Chinese populations are significantly lower than those reported in Western countries. Low BCT rates may now be a concern as they may translate into suboptimal outcomes. A study was undertaken to evaluate BCT rates in a cohort of predominantly Chinese women. Methods. All patients who underwent surgery on the breast at the authors' healthcare facility between October 2008 and December 2011 were included in the study and outcomes of treatment were evaluated. Results. A total of 171 patients were analysed. Two-thirds of the patients were of Chinese ethnicity. One hundred and fifty-six (85.9\%) underwent BCT. Ninety-eight of 114 Chinese women (86\%) underwent BCT. There was no difference in the proportion of women undergoing BCT based on ethnicity. After a median of 49 months of follow-up, three patients (1.8\%) had local recurrence and 5 patients (2.9\%) suffered distant metastasis. Four patients $(2.3 \%)$ have died from their disease. Conclusion. BCT rates exceeding $80 \%$ in a predominantly Chinese population are possible with acceptable local and distant control rates, thereby minimising unnecessary mastectomies.
\end{abstract}

\section{Introduction}

As a result of prospective randomised controlled trials (RCTs) beginning in the 1970s, breast conservation therapy (BCT) was adopted as an appropriate alternative for the treatment of breast cancer and has been an accepted option for more than three decades [1-5]. A consensus statement in 1991 endorsed BCT as the surgical therapy of choice, for it offered similar survival rates while preserving the form of the breast [4]. Recent data suggests that in the presence of modern adjuvant therapies, instead of equivalent survival outcomes, BCT could be superior to mastectomy for the treatment of breast cancer [6-10]. A large retrospective analysis evaluating women with early breast cancer similar to those in one RCT demonstrated a higher 10-year breast-cancer-specific survival for women who had undergone BCT when compared with mastectomy with or without radiation $[2,6]$. For patients who had characteristics unlike those in the RCTs, higher mastectomy rates were found to be associated with poorer survival outcomes [7-9], and, in a prospective series studying hormone-positive tumours, BCT resulted in lower local recurrence rates and improved survival [10].

Despite the longstanding acceptance of BCT, its utilisation in predominantly Chinese communities has been reported to be lower than in Western populations $[6,11-$ 18]. Approximately $75-85 \%$ of women with early stage breast cancer are expected to be candidates for BCT [19], yet BCT rates in predominantly Chinese populations are reported to average 30\%, even for T1-T2 tumours [11-15] (Table 1). An absolute improvement of $4 \%$ in breast-cancer-specific survival rates was reported with BCT rates of $70 \%$ [6]. It was also estimated that, for each 1-percentage-point rise in the mastectomy rate, there would be a concomitant fall in 7-year survival by $0.1 \%$ [7]. It can be inferred from these calculations that, on a population basis, no survival benefit is expected with a mastectomy rate of $70 \%$. There is therefore a pressing need to relook at surgical treatment in predominantly Chinese communities as a persistently 


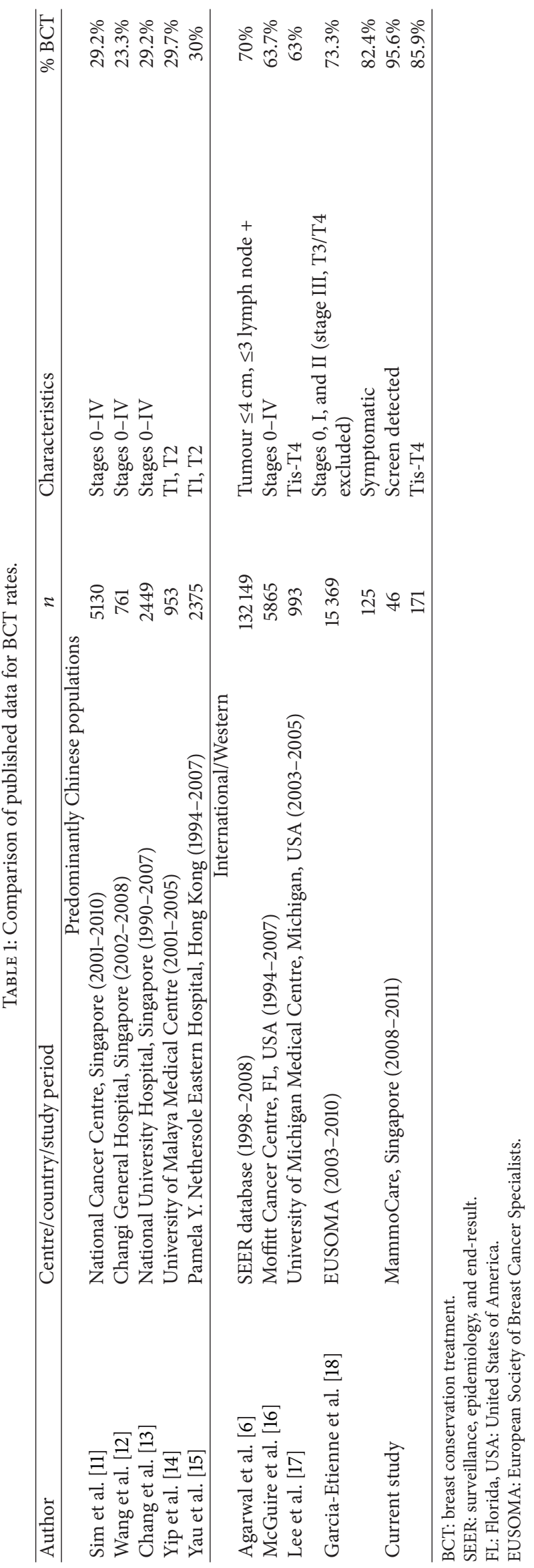


low BCT rate could translate to suboptimal outcomes. The reasons cited for low BCT rates include cultural preferences, surgeon bias, and factors relating to physical attributes [1115]. Chinese women have been shown to have smaller breast tissue volume [20], which poses challenges for good cosmetic outcomes in BCT [21]. This study was therefore performed to review the authors' experience in treating women with BCT in a predominantly Chinese community, evaluate BCT rates in this cohort, and compare it with prior reports.

\section{Materials and Methods}

A retrospective analysis of all patients with breast malignancies who underwent operative treatment by clinicians at this medical facility between October 2008 and December 2011 was performed. Preoperative diagnostic workup consisted of clinical examination and standard imaging with mammography and sonography, and percutaneous needle biopsies were done for diagnosis where possible. In certain clinical settings where percutaneous biopsy was not possible or inconclusive, like where there was insufficient compression thickness or discordant imaging and biopsy results, a surgical diagnostic procedure was performed. Routine magnetic resonance imaging (MRI) was not done.

Following diagnosis, patients deemed eligible for BCT were given the option of an attempt at breast conservation or to proceed directly to mastectomy, with or without reconstruction. Eligibility for BCT was made based on the surgeon's assessment of the ability to achieve a reasonable cosmetic result after tumour resection with clear margins. Multifocal and multicentric breast cancers (MFMCBC) were not considered to be ineligible if preoperative evaluation indicated the possibility of en bloc excision of all foci through a single incision. If the tumour(s) was assessed to be too large, the patient was offered neoadjuvant medical therapy and had placement of radioopaque clip(s) prior to its commencement. These were localised before wide excision, which was guided by the position(s) of the marker clip(s).

Patients who were assessed to be eligible for BCT and who agreed to undergo a trial of BCT had wide excision of their lesion(s) performed through a single incision. Incisions were planned such that they coursed over the lesions where possible. In the instances where there were more than one tumour foci, the incision was sited above at least one of the lesions. If the other lesion(s) were more than $2 \mathrm{~cm}$ away from the incision, radioopaque clips were positioned in the tumour bed for ease of radiotherapy administration. Following tumour extirpation with negative margins, partial mastectomy defects were repaired using local tissue rearrangement techniques only. This was performed by mobilising full thickness parenchymal flaps off the pectoralis fascia, advancing the pillars and directly apposing them with sutures. None of the patients had volume replacement using autologous flaps or implants.

All patients who were intended for BCT had intraoperative frozen section analysis (IFSA) for margins status. If margins were positive at the time of surgery, further margins were excised until proven to be negative. These were reassessed at paraffin sections. Successful BCT of MFMCBC was defined as operative attainment of clear margins (no ink on tumour) [22] and a reasonable cosmetic outcome. In clinical scenarios where this was thought to be unattainable, mastectomy was recommended. Mastectomy was also performed for patients according to preference for breast removal rather than BCT.

The patients were referred both to a medical oncologist and to radiation oncologists for discussions relating to the need for further adjuvant treatment. Patients were considered to have completed therapy if they adhered to recommended treatment regimens. Systemic therapy was given based on the discretion of the treating medical oncologist. Whole breast irradiation was given for women who underwent BCT with a boost to the tumour bed according to the preference of the radiation oncologist. Patients who underwent mastectomy with large tumours, more than 3 positive axillary lymph nodes, and lymphovascular invasion were treated with postmastectomy radiotherapy.

Statistical analyses of the respective associations were performed using SPSS (Chicago, IL) version 11 advanced statistical software module. Comparisons of categorical variables were performed using the chi-squared test. Continuous variables with median or mean values were compared using the Student's $t$-test or Mann-Whitney $U$ test where appropriate.

\section{Results}

A total of 177 female patients were treated during the study period. However, 6 were lost to follow-up before any cancerrelated events were noted, leaving 171 for analysis. Clinicopathological characteristics of the cohort are summarised in Table 2. Of note, 147 patients (86\%) underwent BCT. Twentyfour patients had a mastectomy. Fifteen of these $(62.5 \%)$ had contraindications to BCT based on size and improbability of attaining negative margins without compromising cosmesis, while nine elected to have a mastectomy despite being eligible for BCT. The mean age of these patients who chose a mastectomy was 58 years, which was significantly higher than that of those who underwent BCT, whose mean age was 47.9 years $(P=0.003)$. There was no significant difference between the mean ages of patients who had BCT and those who underwent mastectomy out of necessity (mean age: 50.1 years) based on therapeutic principles $(P=0.40)$. The majority of the cohort was Chinese $(66.7 \%)$ and was generally expected to have smaller volume breast tissue than women of other ethnicities $[15,20]$. There were a fair proportion of Caucasian women and women of other ethnic origins as well, and no difference in the proportion of women undergoing BCT based on ethnicity was demonstrated $(P=0.88)$.

One hundred and fifty-six of the total of 171 (91.2\%) were assessed to be suitable candidates for BCT. However, nine patients of $156(5.8 \%)$ decided against BCT. The main reasons given by these few patients were a perceived superiority of survival with mastectomy and reduction of anxiety relating to follow-up. The mean tumour size for patients who underwent BCT was $19.2 \mathrm{~mm}$, while that for women who elected for mastectomy despite being suitable candidates for BCT was $18.3 \mathrm{~mm}$. There was no significant difference between the two groups $(P=0.83)$. In contrast, there was a significant 
TABLE 2: Summary of demographic, clinicopathologic, and outcome data for study population.

\begin{tabular}{|c|c|c|c|c|c|c|c|c|c|}
\hline \multirow{2}{*}{ Clinicopathologic characteristic } & \multirow{2}{*}{\multicolumn{2}{|c|}{$\begin{array}{r}\text { All patients }(n=171) \\
(\%)\end{array}$}} & \multicolumn{2}{|c|}{$\mathrm{BCT}(n=147)$} & \multicolumn{4}{|c|}{ Mastectomy $(n=24)$} & \multirow{2}{*}{$P$ value } \\
\hline & & & & $(\%)$ & By need (15) & $(\%)$ & By choice (9) & $(\%)$ & \\
\hline \multicolumn{10}{|l|}{ Age in years } \\
\hline Median (range) & $48(28-78)$ & & & & & & & & \\
\hline Mean (SD) & $48.6(10)$ & & $47.9(10)$ & & $50.1(8.8)$ & & & & 0.40 \\
\hline Mean (SD) & & & $47.9(10)$ & & & & $58.0(6.9)$ & & 0.003 \\
\hline Ethnicity & & & & & & & & & 0.88 \\
\hline Chinese & 114 & $(66.7)$ & $98 / 114$ & $(86.0)$ & $8 / 114$ & $(7.0)$ & $8 / 114$ & $(7.0)$ & \\
\hline Malay/Indonesian & 12 & $(7.0)$ & $10 / 12$ & $(83.3)$ & $2 / 12$ & $(16.7)$ & $0 / 12$ & & \\
\hline Indian & 11 & $(6.4)$ & $10 / 11$ & $(91.0)$ & $1 / 11$ & $(9.0)$ & $0 / 12$ & & \\
\hline Other Asian & 14 & $(8.2)$ & $13 / 14$ & $(92.9)$ & $1 / 14$ & $(7.1)$ & $0 / 14$ & & \\
\hline Caucasian & 20 & $(11.7)$ & $16 / 20$ & $(80.0)$ & $3 / 20$ & $(15.0)$ & $1 / 20$ & $(5.0)$ & \\
\hline Mode of presentation & & & & & & & & & 0.07 \\
\hline Symptomatic tumours & 125 & $(73.1)$ & $103 / 125$ & $(82.4)$ & $13 / 125$ & $(10.4)$ & $9 / 125$ & $(7.2)$ & \\
\hline Screen detected lesions & 46 & $(26.9)$ & $44 / 46$ & $(95.6)$ & $2 / 46$ & $(4.3)$ & 0 & & \\
\hline All patients & 171 & & $147 / 171$ & $(85.9)$ & $15 / 171$ & $(8.8)$ & $9 / 171$ & (5.3) & \\
\hline \multicolumn{10}{|l|}{ Tumour size in mm (range) } \\
\hline Median (range) & $19.0(3-97)$ & & $18.0(3-72)$ & & $35.0(4-97)$ & & $15.9(3-35)$ & & \\
\hline Mean (SD) & $21.1(15.4)$ & & $19.2(12.1)$ & & $40.5(28.0)$ & & & & 0.000 \\
\hline (DCIS included) & & & $19.2(12.1)$ & & & & $18.3(12.9)$ & & 0.83 \\
\hline$\leq 20 \mathrm{~mm}$ & 108 & $(63.2)$ & $100 / 108$ & $(92.6)$ & $4 / 108$ & $(3.7)$ & $4 / 108$ & $(3.7)$ & \\
\hline $21-50 \mathrm{~mm}$ & 51 & $(29.8)$ & $39 / 51$ & $(76.5)$ & $7 / 51$ & $(13.7)$ & $4 / 51$ & $(7.8)$ & \\
\hline$>50 \mathrm{~mm}$ & 9 & $(5.3)$ & $6 / 9$ & $(66.7)$ & $3 / 9$ & $(33.3)$ & 0 & & \\
\hline $\mathrm{T} 4$ & 3 & (1.8) & $2 / 3$ & $(66.7)$ & $1 / 3$ & $(33.3)$ & & & \\
\hline Pathologic stage & & & & & & & & & $<0.001$ \\
\hline 0 & 22 & $(12.9)$ & $20 / 22$ & $(90.1)$ & $1 / 22$ & $(4.5)$ & $1 / 22$ & $(4.5)$ & \\
\hline I & 70 & $(41.0)$ & $69 / 70$ & $(98.6)$ & 1 & $(1.4)$ & 0 & & \\
\hline II & 55 & $(32.2)$ & $46 / 55$ & $(83.6)$ & $4 / 55$ & $(7.3)$ & $5 / 55$ & (9.1) & \\
\hline III & 21 & $(12.3)$ & $11 / 221$ & $(52.4)$ & $8 / 21$ & $(38.1)$ & $2 / 21$ & $(9.5)$ & \\
\hline IV & 1 & $(0.6)$ & 0 & & 1 & & & & \\
\hline Unknown & 2 & $(1.2)$ & $1 / 2$ & & & & $1 / 2$ & & \\
\hline Histological type & & & & & & & & & 0.34 \\
\hline DCIS & 22 & $(12.9)$ & $20 / 22$ & $(91.0)$ & $1 / 22$ & $(4.5)$ & $1 / 22$ & $(4.5)$ & \\
\hline Invasive ductal & 132 & $(77.2)$ & $114 / 132$ & $(86.4)$ & $11 / 132$ & (8.3) & $7 / 125$ & (5.6) & \\
\hline Invasive lobular & 7 & $(4.1)$ & $5 / 7$ & $(71.4)$ & $1 / 7$ & $(1.4)$ & $1 / 7$ & $(1.4)$ & \\
\hline Other invasive & 10 & (5.8) & $8 / 10$ & $(80.0)$ & $2 / 10$ & $(20)$ & & & \\
\hline Grade & & & & & & & & & 0.48 \\
\hline DCIS & 22 & $(12.9)$ & $20 / 22$ & $(91.0)$ & $1 / 22$ & $(4.5)$ & $1 / 22$ & $(4.5)$ & \\
\hline 1 & 29 & $(17.0)$ & $28 / 29$ & $(96.5)$ & $1 / 29$ & (3.6) & 0 & & \\
\hline 2 & 61 & $(35.6)$ & $50 / 61$ & $(81.7)$ & $6 / 61$ & $(9.8)$ & $5 / 61$ & $(8.2)$ & \\
\hline 3 & 54 & $(31.6)$ & $44 / 54$ & $(81.5)$ & $7 / 54$ & $(13.0)$ & $3 / 54$ & $(5.6)$ & \\
\hline Unknown & 5 & $(2.9)$ & $4 / 4$ & $(100)$ & & & & & \\
\hline Neoadjuvant medical therapy & & & & & & & & & $<0.001$ \\
\hline Yes & 25 & $(14.6)$ & $16 / 25$ & $(64.0)$ & $8 / 25$ & $(32.0)$ & $1 / 25$ & $(4.0)$ & \\
\hline No & 146 & $(85.4)$ & $131 / 146$ & $(89.7)$ & $7 / 146$ & $(4.8)$ & $8 / 146$ & $(4.8)$ & \\
\hline Disease extent & & & & & & & & & 0.97 \\
\hline Unifocal & 128 & $(74.6)$ & $110 / 128$ & $(85.9)$ & $11 / 128$ & $(8.6)$ & $7 / 128$ & $(5.5)$ & \\
\hline Multiple foci at diagnosis & 43 & $(25.1)$ & $34 / 40$ & $(85)$ & $4 / 40$ & $(10)$ & $2 / 40$ & $(5)$ & \\
\hline
\end{tabular}

BCT: breast conservation surgery; SD: standard deviation; DCIS: ductal carcinoma in situ. 
difference in the mean tumour sizes of women who had BCT and those who were advised to undergo a mastectomy, the latter group of which had a mean tumour size of $40.5 \mathrm{~mm}$ $(P<0.001)$.

Four patients in the cohort $(2.3 \%)$ required a second therapeutic surgical procedure, three for undetected multicentric tumours seen on postoperative imaging and a fourth for a falsely negative sentinel lymph node (SLN) at IFSA. The last patient had multifocal disease with two separate foci of invasive ductal carcinoma, grade III, $35 \mathrm{~mm}$ and $8 \mathrm{~mm}$, and 2 of 22 lymph nodes involved. All four patients who needed reoperation had multifocal or multicentric breast cancers (MFMCBC) and are currently disease-free. The mean pathologic tumour size for those requiring reoperation was $32.3 \mathrm{~mm}$, while those who had a single operation had a mean tumour size of $20.8 \mathrm{~mm}(P=0.14)$. There were no patients who required a reexcision on the basis of false negative margins at IFSA.

The median follow-up period was 49 months (range 21-68 months). None of the 22 patients who presented with ductal carcinoma in situ only developed locoregional recurrence or distant metastasis. Of the other 149 patients with invasive carcinoma, two patients who underwent BCT developed local failure (1.4\%). A total of four patients with invasive disease, two who had undergone BCT and two mastectomy, developed distant disease and have succumbed to their disease (2.3\%). A summary of locoregional and distant events is given in Table 3.

\section{Discussion}

The last two decades of the twentieth century witnessed a paradigm shift of treatment concepts for breast cancer, with definitive evidence from prospective randomised trials demonstrating that performing less surgery resulted in equivalent survival outcomes. This led to the establishment of BCT and a steady increase in its utilisation. Unexpectedly, in the last decade, a rising trend of mastectomy rates has been observed in some Western communities [16, 23]. Despite this rise, mastectomy rates for early breast cancer in these countries are still lower than the high rates reported in predominantly Chinese populations. Mastectomy rates need to be reexamined in the presence of contemporary reports indicating possible higher cancer-specific survival, lower surgical complication rates, cost-effectiveness of treatment, and improved quality of life outcomes with BCT [6-10, 21, 24-28]. This is juxtaposed against the possible psychological benefit that mastectomy and contralateral prophylactic mastectomy (CPM) offer [24,29], but the diminution of anxiety might not completely compensate for poorer quality of life outcomes with longer breast cancer survivorship $[26,30]$.

Specifically for predominantly Chinese populations, reasons cited for low BCT rates include cultural preferences, surgeon bias, and factors relating to physical attributes [1115]. Addressing the issue of physical attributes first, Chinese women tend to have smaller volume breast (SVB) tissue which may pose a barrier to BCT $[15,20]$. In this series, overall BCT rate was $85.9 \%$. Among Chinese women, who formed two-thirds of the cohort, BCT rate was $86 \%$. There was no significant difference of the proportion of Chinese women undergoing BCT compared with women of other ethnic groups. In particular, $80 \%$ of Caucasian women had BCT. This rate is similar to other reported series in a Western context (Table 1). This data appears to suggest that the physical attributes of Chinese women may not have a significant impact on BCT rates. In a study by Collins et al., of 125 women who were eligible for either BCT or mastectomy, $35 \%$ elected to have a mastectomy [31]. In the present study, of 156 patients who were considered eligible for BCT or mastectomy, only 5.8\% decided against breast conservation. This data suggests that the local Chinese culture may be a factor in favour of BCT, rather than a condition in support of high mastectomy rates.

Another factor influencing patient decision for mastectomy is surgeon's advice [32]. In this study, 156 of 171 patients $(91.2 \%)$ were assessed preoperatively to be suitable candidates for BCT. Nine patients decided in favour of mastectomy, making utilisation of BCT in this cohort $94.2 \%$. Eligible patients who decided for mastectomy had a mean tumour size not significantly different from those who underwent successful BCT. Hence, it is reasonable to conclude that successful BCT would have been possible for this group of women, suggesting that, on the whole, eligibility of Chinese women for BCT is not significantly different from women of other ethnic origins. The difference perhaps is surgeon philosophy, which may account for varying reported mastectomy rates in different geographical locations [23, 33]. Surgeons with a strong bias toward mastectomy will require clinical circumstances extremely favourable for BCT before contemplating it, while surgeons whose default position is conservative surgery would be more inclined to explore innovative methods of achieving BCT even in challenging clinical situations. The development of oncoplastic breast surgery was likely the result of this creative pressure. There are several categories of techniques that fall into this broad description. The authors prefer the use of only volume displacement or local tissue rearrangement techniques with direct apposition of adequately mobilised residual uninvolved parenchymal pillars with sutures. This approach results in better patient satisfaction than mastopexy and avoids the issues with surgical clip migration with the use of mammoplasty techniques which require extensive tissue mobilization [21, 34, 35]. Moreover, local tissue rearrangement techniques result in lower complication rates and superior cosmetic outcomes compared to complex reconstructive techniques [21].

Although the presence of multicentric disease, or MFM$\mathrm{CBC}$, is a conventional contraindication for BCT [36], a recent expert consensus considers this scenario a relative rather than an absolute contraindication [37]. MFMCBC pose technical challenges for BCT but may be surmounted with careful attention to surgical planning and technique. Patients initially considered ineligible for BCT by some surgeons might in fact be suitable candidates once appropriate measures are applied, avoiding "unnecessary mastectomies." Examples of these situations are depicted in Figures 1 and 2. Based on imaging findings and percutaneous biopsy results, these two patients were recommended to have a mastectomy at another tertiary referral cancer centre. A second 


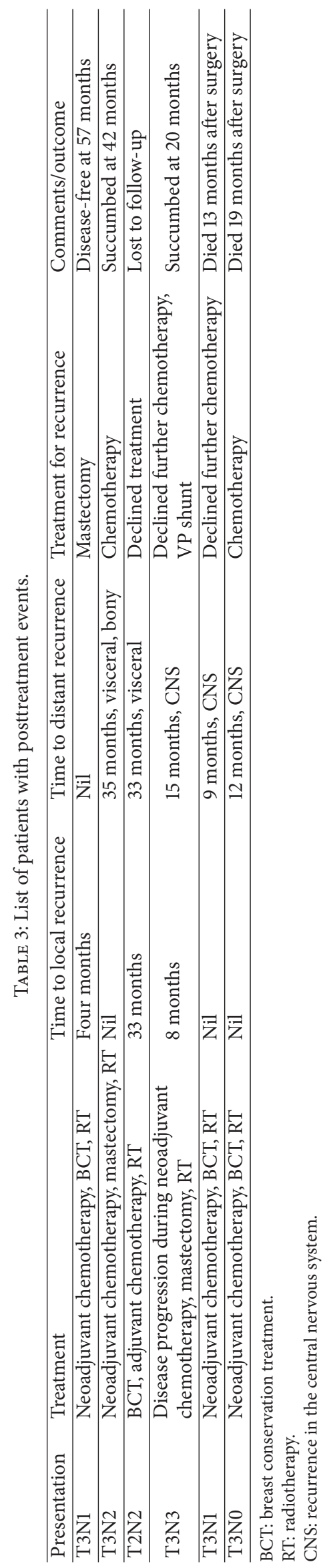




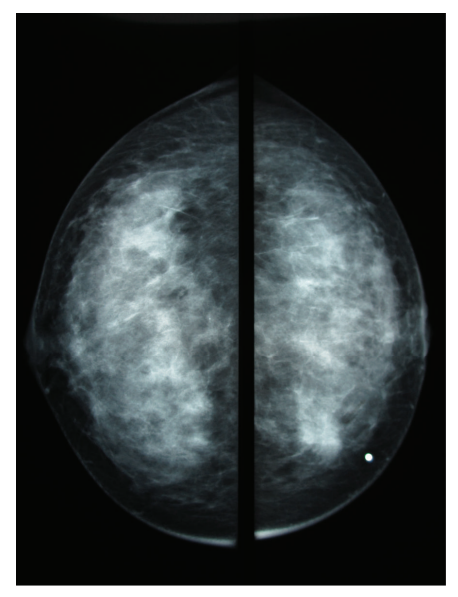

(a)

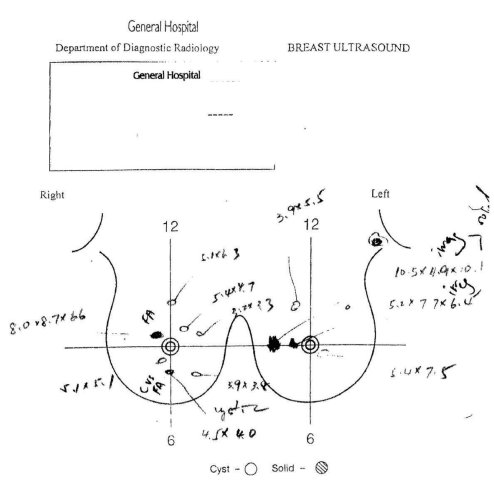

(c)

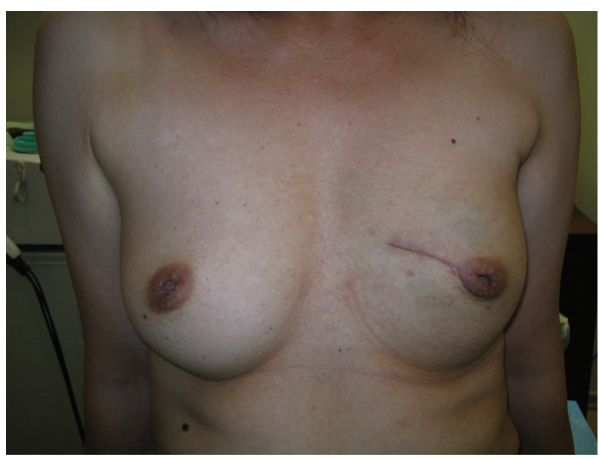

(e)

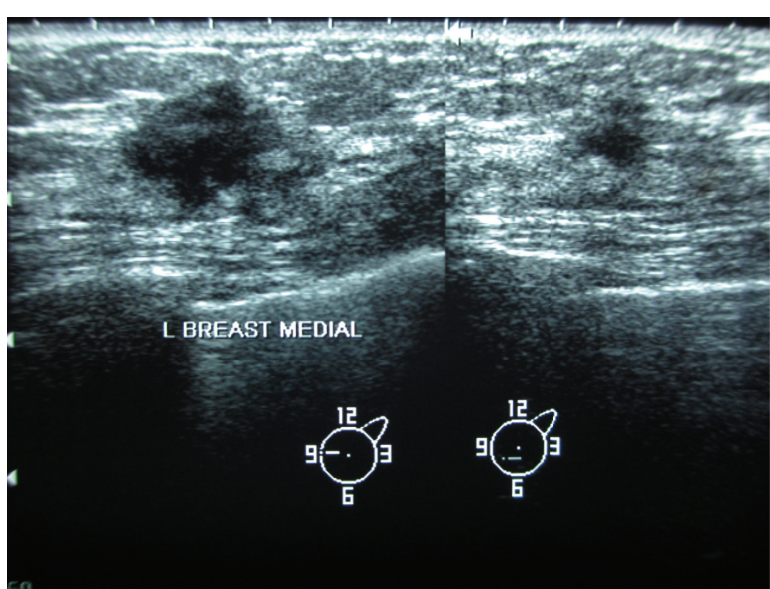

(b)

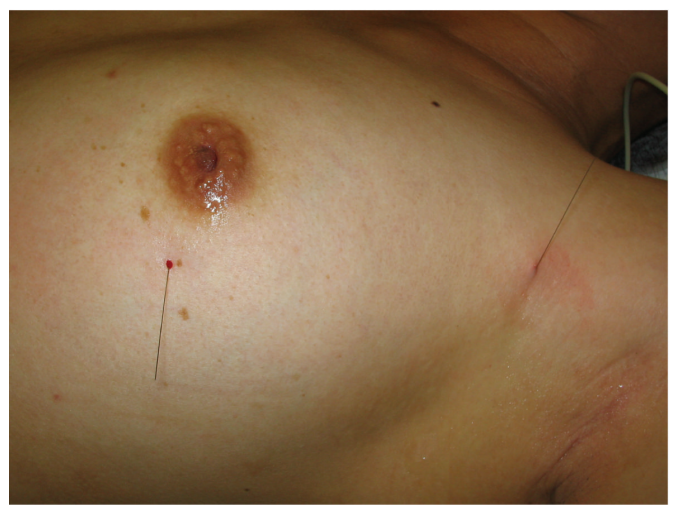

(d)

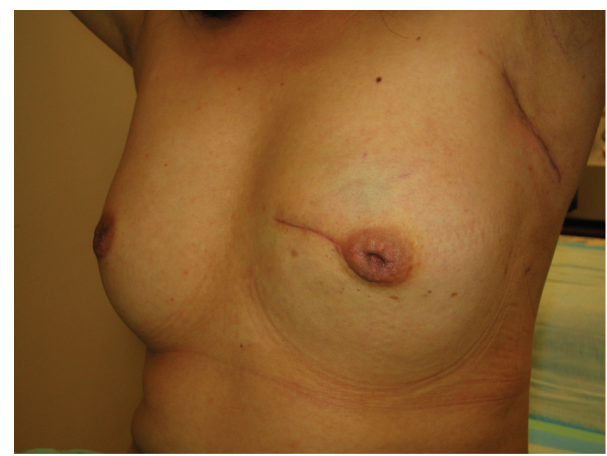

(f)

FIGURE 1: ((a)-(f)) Avoiding mastectomy in a patient with "multifocal tumour" on imaging. This 45-year-old patient was diagnosed with what was thought to be multifocal invasive ductal carcinoma at another facility following core biopsy. Mastectomy was originally recommended at the first centre due to the presence of multiple synchronous ipsilateral tumours and proximity of one lesion to the nipple. She sought a second opinion at the authors' facility and was agreeable to a "trial of breast conservation treatment." Localisation of the impalpable periareolar lesion and of the suspicious axillary lymph node was performed. She underwent an en bloc wide excision of the two left breast lesions through a boomerang incision and axillary staging through a separate axillary incision. The sentinel node coincided with the localised node and was found to be positive for metastasis on frozen section analysis. She underwent axillary dissection at the same operation. Histology was reported as a $4 \mathrm{~cm}$ invasive ductal carcinoma, with no intervening normal tissue between the clinical lesions. Three of sixteen axillary lymph nodes were involved. She is currently disease-free after more than 5 years.

opinion was sought with the authors and they were willing to undergo a "trial of BCT." Both underwent successful BCT and are now disease-free more than five years after surgical treatment. Sometimes, approaches which contravene conventional guideline recommendations are necessary. For example, unlike guidelines which recommend skin crease incisions [36], radial incisions may be necessary to incorporate larger lesions, multiple tumours, and allow adequate 


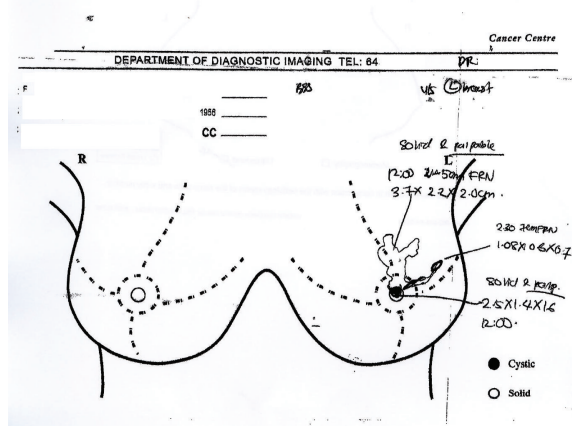

(a)

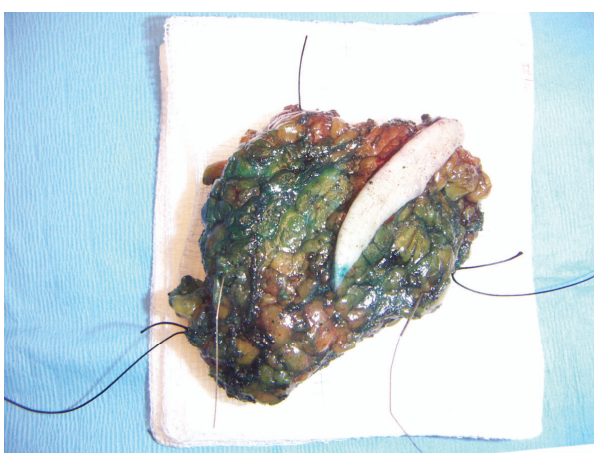

(c)

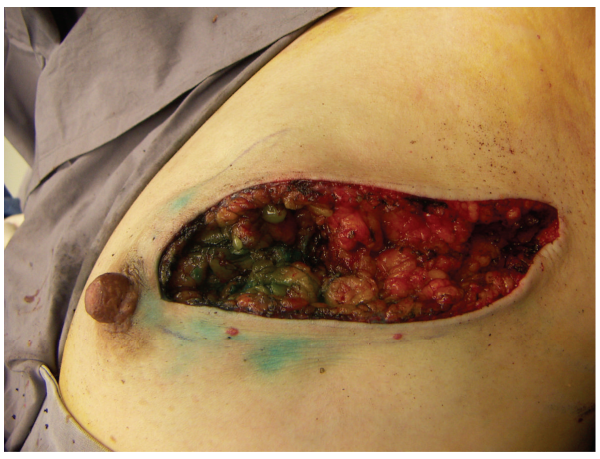

(e)

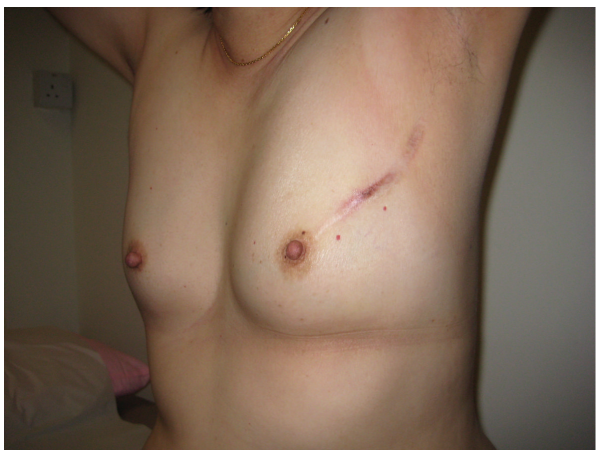

(g)

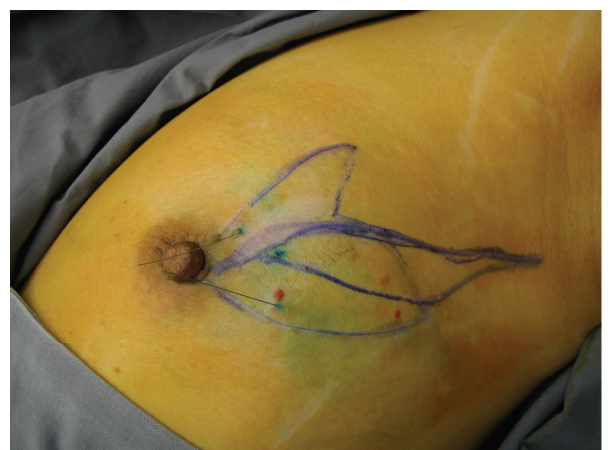

(b)

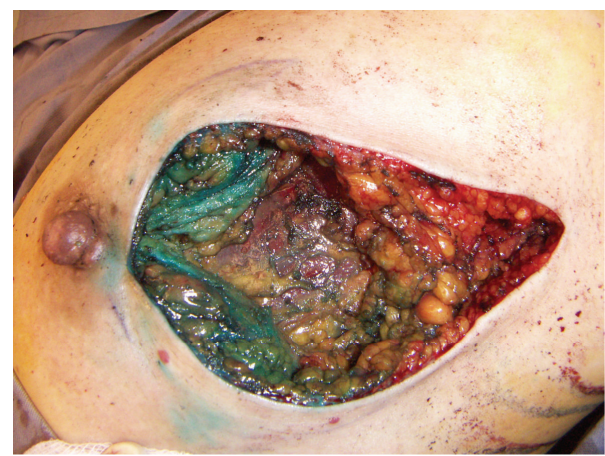

(d)

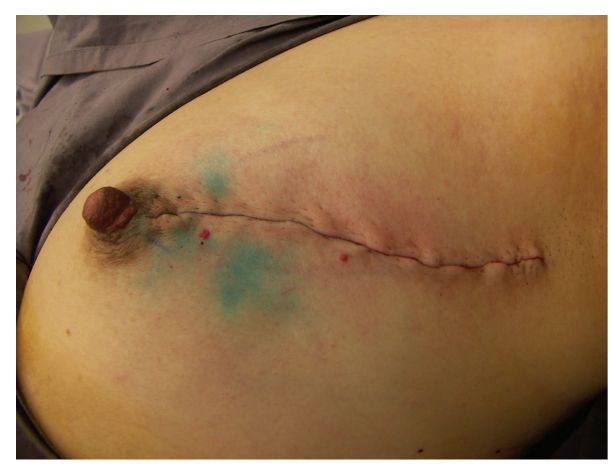

(f)

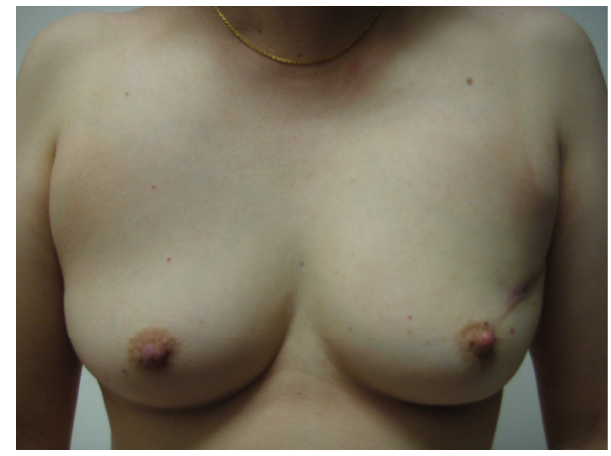

(h)

FIgURE 2: ((a)-(h)) Avoiding mastectomy in a patient with "multicentric tumour" on imaging. This patient was diagnosed to have high grade ductal carcinoma in situ (DCIS) at an oncology centre and was offered mastectomy on the assumption that this was a multicentric lesion. She sought a second opinion with the authors and was agreeable to a "trial of breast conservation treatment." Just prior to surgery, the lateral and medial extents of her dual-segment disease were localised under ultrasound guidance. Tissue resection was planned as indicated to balance the need for negative margins and retention of sufficient uninvolved parenchyma for defect repair. Through a radial incision and eccentric ellipse, an en bloc resection of the lesion using a multisegment resection pattern was performed. Sentinel node biopsy was performed through the same incision for this palpable high grade DCIS. Histology was reported as a unifocal $25 \mathrm{~mm}$ high grade DCIS. No multicentric component could be identified. She completed all adjuvant treatment and is now disease-free more than 5 years after surgery. 
exposure for remodelling to avoid deformity [38, 39] (Figures 1 and 2).

Advances in imaging resulted in an improved ability to identify multiple tumour foci. However, recent data suggest that this increase in identification of MFMCBC with MRI results in higher mastectomy rates without definitive improvement in survival outcomes [40,41]. Hence, a selective approach should be taken with the use of preoperative MRI. In this study, because preoperative MRI was not used, four patients, all of whom had multiple ipsilateral cancers, required a reoperation. MRI may be applied when multiple cancers or extensive microcalcifications are detected on conventional imaging to avoid reoperations. However, further work may be necessary in this area to assess specific selection criteria for its use to balance this need against a potential for increasing mastectomy use in this group of patients.

The reported rise in mastectomy rates is observed with a concomitant increase in the use of contralateral prophylactic mastectomy (CPM) [24, 25]. Factors contributing to this phenomenon include the use of preoperative MRI, the presence of multicentric disease, the availability of reconstructive surgery, fear of cancer recurrence, concerns over the risk of contralateral breast cancer (CBC), and a perceived survival benefit with mastectomy [24, 29]. The first three factors, which may also influence high mastectomy rates in Chinese populations, are clinician related and have been discussed earlier. The latter three involve patient psychology and information dissemination for decision-making. Data on how communication methods and decision aids affect patient's surgical decisions is varied [31-33,42]. It is interesting to note that BCT tends to be preferred in women without breast cancer [43], but once diagnosed, higher mastectomy rates as the definitive operation were reported with greater patient involvement in decision-making [44]. In the light of recent data that higher mastectomy rates could lead to poorer survival outcomes, a reexamination of patient decision-making processes is needed. One factor which may influence patient choice in favour of mastectomy despite eligibility for BCT is the availability of reconstruction [24]. While reconstruction does lead to improved body image [26], its availability prior to a "trial of BCT" may have an adverse effect on $\mathrm{BCT}$ rates, possibly encouraging not only mastectomy but CPM as well. Some authors have concluded that, based on current evidence, the use of CPM for the purposes of risk reduction in sporadic breast cancer is unjustified $[25,44]$. Other investigators share a different opinion [24]. Arriving at the delicate balance between a paternalistic approach recommending treatment associated with evidence-based superior outcomes and allowing patient autonomy in decision-making is complex and elusive. This difficulty can make a "simple can be harder than complex" situation in the pursuit of improvements in BCT [19]. Further work on this subject is warranted.

In a surgical era where there is increasing public acceptance towards less invasive procedures for equivalent outcomes in breast cancer, as has been seen in the paradigm shifts with percutaneous breast biopsies and the management of the axilla, it is perplexing that BCT rates are persistently low in predominantly Chinese communities, and mastectomy and CPM rates are rising in other populations. Recent data showing the potential for improved survival, lower complication rates, cost-effectiveness, and better body image during survivorship with BCT $[6-10,21,25,26]$ behoves clinicians to consider means of increasing its utilisation. The surgeon factor has been shown to affect BCT rates $[32,45,46]$. The data in this study appears to support this conclusion. It may be reasonable to surmise that surgeons recommending BCT apply operative techniques to surmount patient's physical attributes of SVB. Careful attention to clinical approaches and surgical technique may raise BCT rates in a predominantly Chinese community from approximately $30 \%$ to $86 \%$, minimising unnecessary mastectomies in this population. Further work is needed to investigate if the concepts for improving utilisation of BCT in a population with prevailing low BCT rates may be applied in other settings to reverse a trend of rising mastectomy rates.

The retrospective nature of this study and small cohort are limitations to this study. In addition, the authors' practice in a private healthcare facility may serve as a selection bias where women who actively sought BCT were treated at the authors' facility. Notwithstanding, the distribution of tumour sizes is not dissimilar from other reports where oncoplastic reduction mammoplasty was performed [34,35], indicating that the concepts discussed herein may be applicable to other healthcare settings to raise $\mathrm{BCT}$ rates.

\section{Conclusion}

Higher BCT rates demonstrated in this study than previously reported for predominantly Chinese communities suggest that it is possible to minimise unnecessary mastectomies in this select population. Further work is needed to define modifying factors.

\section{Conflict of Interests}

The authors have no conflict of interests to declare.

\section{References}

[1] U. Veronesi, N. Cascinelli, L. Mariani et al., "Twenty-year follow-up of a randomized study comparing breast-conserving surgery with radical mastectomy for early breast cancer," New England Journal of Medicine, vol. 347, no. 16, pp. 1227-1232, 2002.

[2] B. Fisher, S. Anderson, J. Bryant et al., "Twenty-year followup of a randomized trial comparing total mastectomy, lumpectomy, and lumpectomy plus irradiation for the treatment of invasive breast cancer," The New England Journal of Medicine, vol. 347, no. 16, pp. 1233-1241, 2002.

[3] M. M. Poggi, D. N. Danforth, L. C. Sciuto et al., "Eighteenyear results in the treatment of early breast carcinoma with mastectomy versus breast conservation therapy," Cancer, vol. 98, no. 4, pp. 697-702, 2003.

[4] "NIH consensus conference. Treatment of early-stage breast cancer," The Journal of the American Medical Association, vol. 265, no. 3, pp. 391-395, 1991.

[5] S. A. McLaughlin, "Surgical management of the breast: breast conservation therapy and mastectomy," Surgical Clinics of North America, vol. 93, no. 2, pp. 411-428, 2013. 
[6] S. Agarwal, L. Pappas, L. Neumayer, K. Kokeny, and J. Agarwal, "Effect of breast conservation therapy vs mastectomy on disease-specific survival for early-stage breast cancer," JAMA Surgery, vol. 149, no. 3, pp. 267-274, 2014.

[7] J. M. Brooks, E. A. Chrischilles, M. B. Landrum et al., "Survival implications associated with variation in mastectomy rates for early-staged breast cancer," International Journal of Surgical Oncology, vol. 2012, Article ID 127854, 9 pages, 2012.

[8] N. L. Keating, M. B. Landrum, J. M. Brooks et al., "Outcomes following local therapy for early-stage breast cancer in non-trial populations," Breast Cancer Research and Treatment, vol. 125, no. 3, pp. 803-813, 2011.

[9] M. A. Schonberg, E. R. Marcantonio, D. Li, R. A. Silliman, L. Ngo, and E. P. McCarthy, "Breast cancer among the oldest old: tumor characteristics, treatment choices, and survival," Journal of Clinical Oncology, vol. 28, no. 12, pp. 2038-2045, 2010.

[10] M. van Hezewijk, E. Bastiaannet, H. Putter et al., "Effect of local therapy on locoregional recurrence in postmenopausal women with breast cancer in the Tamoxifen Exemestane Adjuvant Multinational (TEAM) trial," Radiotherapy \& Oncology, vol. 108, no. 2, pp. 190-196, 2013.

[11] Y. Sim, V. K. M. Tan, G. H. Ho et al., "Contralateral prophylactic mastectomy in an Asian population: a single institution review," Breast, vol. 23, no. 1, pp. 56-62, 2013.

[12] W. V. Wang, S. M. Tan, and W. L. Chow, "The impact of mammographic breast cancer screening in Singapore: a comparison between screen-detected and symptomatic women," Asian Pacific Journal of Cancer Prevention, vol. 12, no. 10, pp. 2735-2740, 2011.

[13] G. Chang, C. W. Chan, and M. Hartman, "A commentary on delayed presentation of breast cancer in Singapore," Asian Pacific Journal of Cancer Prevention, vol. 12, no. 6, pp. 1635-1639, 2011.

[14] C. H. Yip, N. A. Taib, G. H. Tan, K. L. Ng, B. K. Yoong, and W. Y. Choo, "Predictors of axillary lymph node metastases in breast cancer: is there a role for minimal axillary surgery?" World Journal of Surgery, vol. 33, no. 1, pp. 54-57, 2009.

[15] T.-K. Yau, I. S. Soong, H. Sze et al., "Trends and patterns of breast conservation treatment in Hong Kong: 1994-2007," International Journal of Radiation Oncology, Biology, Physics, vol. 74, no. 1, pp. 98-103, 2009.

[16] K. P. McGuire, A. A. Santillan, P. Kaur et al., "Are mastectomies on the rise? A 13-year trend analysis of the selection of mastectomy versus breast conservation therapy in 5865 patients," Annals of Surgical Oncology, vol. 16, no. 10, pp. 2682-2690, 2009.

[17] M. C. Lee, K. Rogers, K. Griffith et al., "Determinants of breast conservation rates: reasons for mastectomy at a Comprehensive Cancer Center," Breast Journal, vol. 15, no. 1, pp. 34-40, 2009.

[18] C. A. Garcia-Etienne, M. Tomatis, J. Heil et al., "Mastectomy trends for early-stage breast cancer: a report from the EUSOMA multi-institutional European database," European Journal of Cancer, vol. 48, no. 13, pp. 1947-1956, 2012.

[19] I. T. Rubio, "Breast conservative surgery in breast cancer: simple can be harder than complex," Journal of Surgical Oncology, vol. 110, no. 1, p. 1, 2014.

[20] Q. Qiao, G. Zhou, and Y.-C. Ling, "Breast volume measurement in young Chinese women and clinical applications," Aesthetic Plastic Surgery, vol. 21, no. 5, pp. 362-368, 1997.

[21] S. J. Kronowitz, J. A. Feledy, K. K. Hunt et al., "Determining the optimal approach to breast reconstruction after partial mastectomy," Plastic and Reconstructive Surgery, vol. 117, no. 1, pp. 1-11, 2006.

[22] M. S. Moran, S. J. Schnitt, A. E. Giuliano et al., "Society of surgical oncology-American Society for Radiation Oncology consensus guideline on margins for breast-conserving surgery with whole-breast irradiation in stages I and II invasive breast cancer," Annals of Surgical Oncology, vol. 21, no. 3, pp. 704-716, 2014.

[23] A. E. Dragun, B. Huang, T. C. Tucker, and W. J. Spanos, "Increasing mastectomy rates among all age groups for early stage breast cancer: a 10-year study of surgical choice," Breast Journal, vol. 18, no. 4, pp. 318-325, 2012.

[24] A. Soran, A. K. Polat, R. Johnson, and K. P. McGuire, "Increasing trend of contralateral prophylactic mastectomy: what are the factors behind this phenomenon?" Surgeon, vol. 12, no. 6, pp. 316-322, 2014.

[25] A. Roberts, M. Habibi, and K. D. Frick, "Cost-effectiveness of contralateral prophylactic mastectomy for prevention of contralateral breast cancer," Annals of Surgical Oncology, vol. 21, no. 7, pp. 2209-2217, 2014.

[26] S.-Y. Fang, B.-C. Shu, and Y.-J. Chang, "The effect of breast reconstruction surgery on body image among women after mastectomy: a meta-analysis," Breast Cancer Research and Treatment, vol. 137, no. 1, pp. 13-21, 2013.

[27] J. Engel, J. Kerr, A. Schlesinger-Raab, H. Sauer, and D. Hölzel, "Quality of life following breast-conserving therapy or mastectomy: results of a 5-year prospective study," Breast Journal, vol. 10, no. 3, pp. 223-231, 2004.

[28] M. C. Lee, R. S. Bhati, E. E. von Rottenthaler et al., "Therapy choices and quality of life in young breast cancer survivors: a short-term follow-up," The American Journal of Surgery, vol. 206, no. 5, pp. 625-631, 2013.

[29] C. S. Fisher, T. Martin-Dunlap, M. B. Ruppel, F. Gao, J. Atkins, and J. A. Margenthaler, "Fear of recurrence and perceived survival benefit are primary motivators for choosing mastectomy over breast-conservation therapy regardless of age," Annals of Surgical Oncology, vol. 19, no. 10, pp. 3246-3250, 2012.

[30] N. L. Stout, J. M. Binkley, K. H. Schmitz et al., "A prospective surveillance model for rehabilitation for women with breast cancer," Cancer, vol. 118, no. 8, pp. 2191-2200, 2012.

[31] E. D. Collins, C. P. Moore, K. F. Clay et al., "Can women with early-stage breast cancer make an informed decision for mastectomy?" Journal of Clinical Oncology, vol. 27, no. 4, pp. 519-525, 2009.

[32] T. T. Fancher, J. A. Palesty, R. Thomas et al., "A woman's influence to choose mastectomy as treatment for breast cancer," Journal of Surgical Research, vol. 153, no. 1, pp. 128-131, 2009.

[33] R. S. Ballinger, K. F. Mayer, G. Lawrence, and L. Fallowfield, "Patients' decision-making in a UK specialist centre with high mastectomy rates," Breast, vol. 17, no. 6, pp. 574-579, 2008.

[34] B. R. Eaton, A. Losken, D. Okwan-Duodu et al., "Local recurrence patterns in breast cancer patients treated with oncoplastic reduction mammaplasty and radiotherapy," Annals of Surgical Oncology, vol. 21, no. 1, pp. 93-99, 2014.

[35] C. Eichler, M. Kolsch, A. Sauerwald, A. Bach, O. Gluz, and M. Warm, "Lumpectomy versus mastopexy-a post-surgery patient survey," Anticancer Research, vol. 33, no. 2, pp. 731-736, 2013.

[36] M. Morrow, E. A. Strom, L. W. Bassett et al., "Standard for breast conservation therapy in the management of invasive breast 
carcinoma," CA Cancer Journal for Clinicians, vol. 52, no. 5, pp. 277-300, 2002.

[37] A. Goldhirsch, E. P. Winer, A. S. Coates et al., "Personalizing the treatment of women with early breast cancer: highlights of the st gallen international expert consensus on the primary therapy of early breast Cancer 2013," Annals of Oncology, vol. 24, no. 9, pp. 2206-2223, 2013.

[38] M. P. Tan, "The boomerang incision for periareolar breast malignancies," The American Journal of Surgery, vol. 194, no. 5, pp. 690-693, 2007.

[39] M. Tan and O. Ung, "Alternative approaches for oncoplastic breast surgery," Annals of Surgical Oncology, vol. 18, no. 1, pp. 297-299, 2011.

[40] N. Houssami, R. Turner, and M. Morrow, "Preoperative magnetic resonance imaging in breast cancer," Annals of Surgery, vol. 257, no. 2, pp. 249-255, 2013.

[41] A. Fancellu, D. Soro, P. Castiglia et al., "Usefulness of magnetic resonance in patients with invasive cancer eligible for breast conservation: a comparative study," Clinical Breast Cancer, vol. 14, no. 2, pp. 114-121, 2014.

[42] S. Molenaar, M. A. G. Sprangers, E. J. Rutgers et al., "Decision support for patients with early-stage breast cancer: effects of an interactive breast cancer CDROM on treatment decision, satisfaction, and quality of life," Journal of Clinical Oncology, vol. 19, no. 6, pp. 1676-1687, 2001.

[43] D. Lazovich, K. K. Raab, J. G. Gurney, and H. Chen, "Knowledge and preference for breast conservation therapy among women without breast cancer," Women's Health Issues, vol. 10, no. 4, pp. 210-216, 2000.

[44] S. J. Katz, P. M. Lantz, N. K. Janz et al., "Patient involvement in surgery treatment decisions for breast cancer," Journal of Clinical Oncology, vol. 23, no. 24, pp. 5526-5533, 2005.

[45] H. S. Fiegelson, T. A. James, R. M. Single et al., "Factors associated with the frequency of initial total mastectomy: results of a multi-institutional study," Journal of the American College of Surgeons, vol. 216, pp. 966-975, 2013.

[46] Y. Y. Woon and M. Y. P. Chan, "Breast conservation surgerythe surgeon's factor,” The Breast, vol. 14, no. 2, pp. 131-135, 2005. 


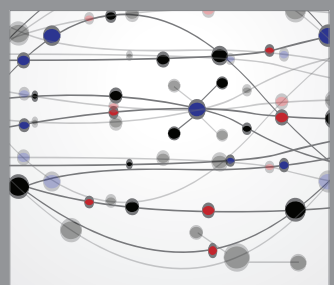

The Scientific World Journal
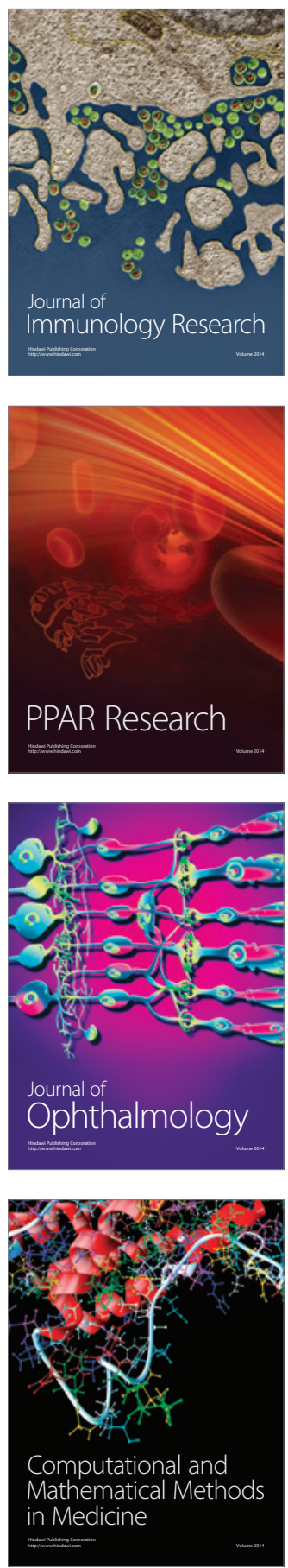

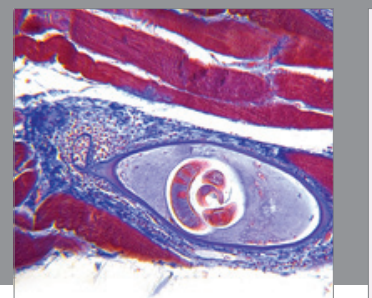

Gastroenterology

Research and Practice
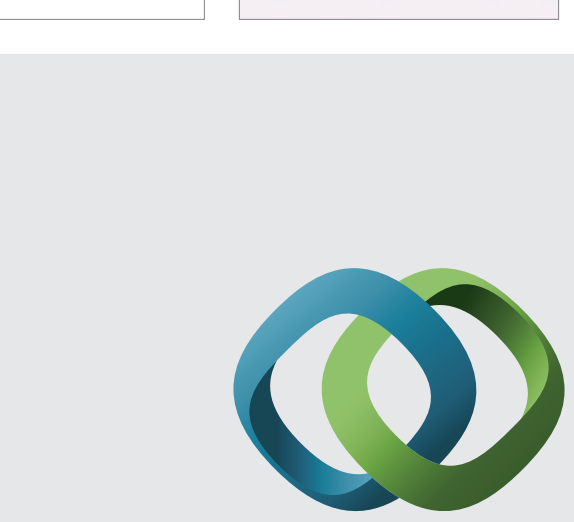

\section{Hindawi}

Submit your manuscripts at

http://www.hindawi.com
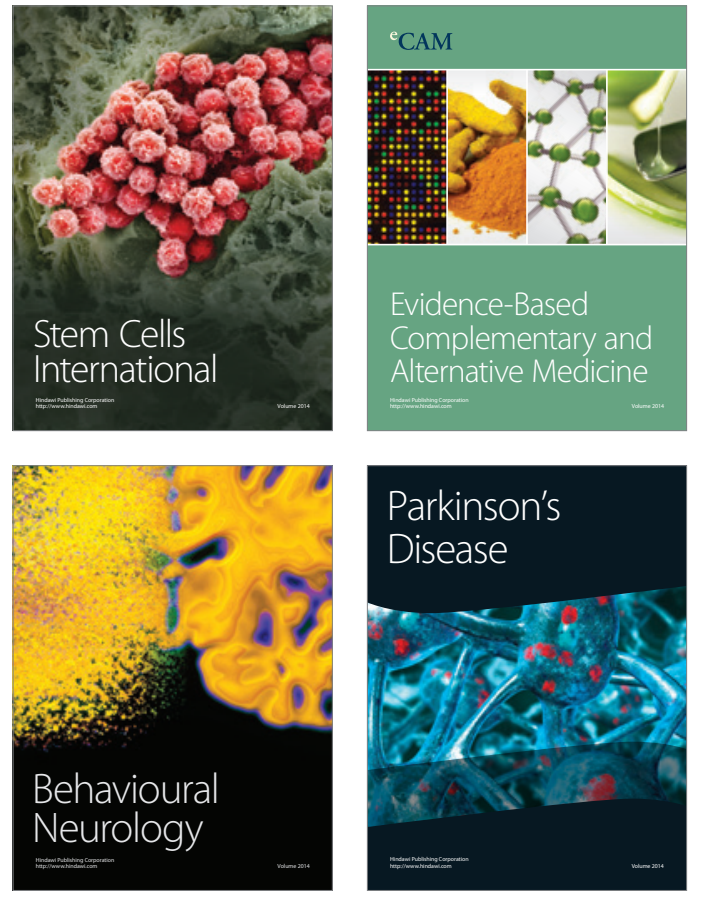
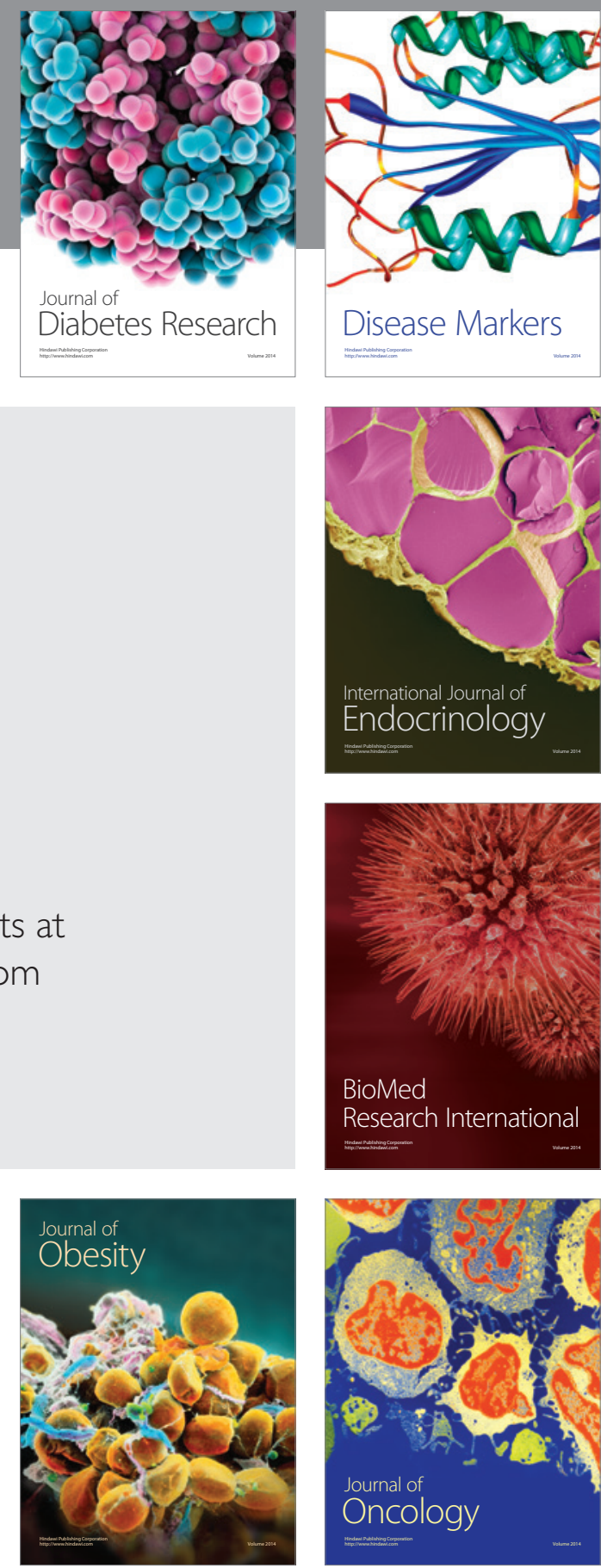

Disease Markers
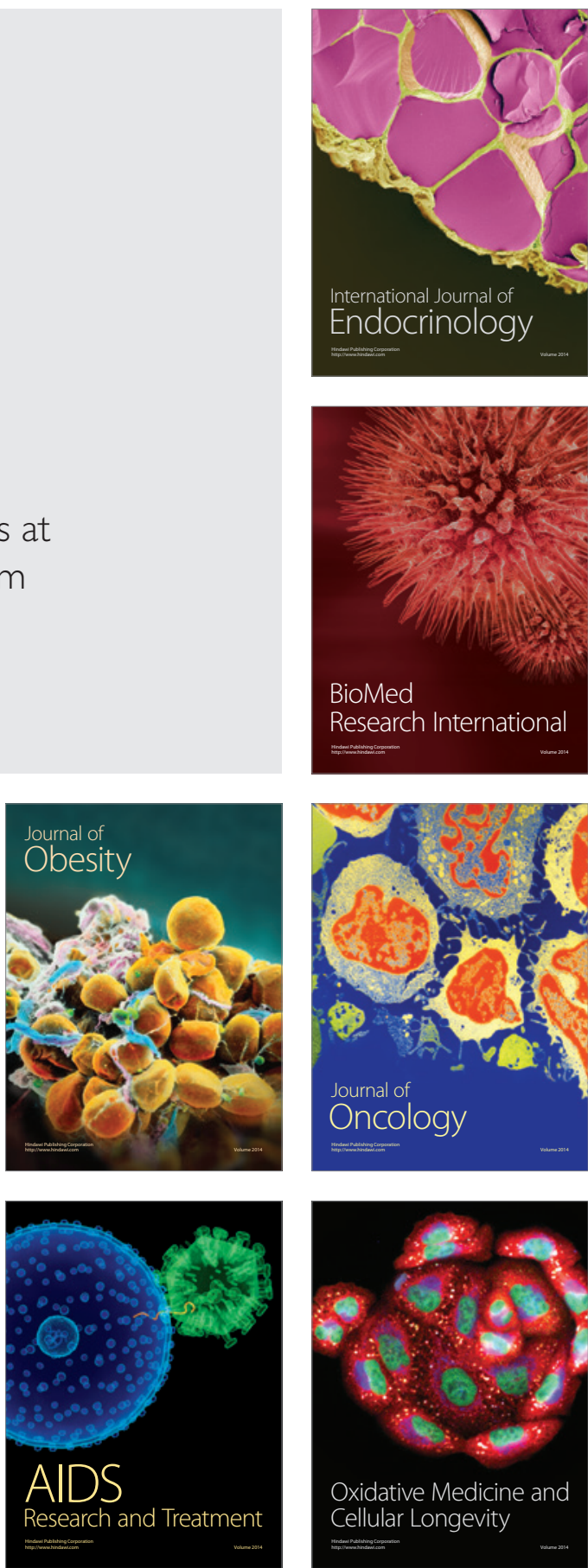